\title{
ESSENTIAL COORDINATE COMPONENTS OF CHARACTERISTIC VARIETIES
}

\author{
ENRIQUE ARTAL BARTOLO*, JORGE CARMONA RUBER ${ }^{\dagger}$, AND JOSÉ IGNACIO \\ COGOLLUDO AGUSTÍN*
}

\begin{abstract}
In this note we give an algebraic and topological interpretation of essential coordinate components of characteristic varieties and illustrate their importance with an example.
\end{abstract}

\section{INTRODUCTION}

Let $\mathcal{C}=\mathcal{C}_{0} \cup \mathcal{C}_{1} \cup \cdots \cup \mathcal{C}_{r}$ be a projective algebraic curve in $\mathbb{P}^{2}$. For convenience, we assume $\mathcal{C}_{0}$ to be a line intersecting the curve $\mathcal{C}^{\prime}:=\mathcal{C}_{1} \cup \cdots \cup \mathcal{C}_{r}$ transversally. In this context, $\mathbb{C}^{2}$ will refer to $\mathbb{P}^{2} \backslash \mathcal{C}_{0}$. Let $X:=\mathbb{P}^{2} \backslash \mathcal{C}$ denote its complement and $\tilde{X}$ the universal abelian cover of $X$. The group of covering transformations of $\tilde{X} \rightarrow X$ acts on $H_{1}(\tilde{X} ; \mathbb{Z})$ and endows it with a $\Lambda_{\mathcal{C}}^{\mathbb{Z}}$-module structure, where $\Lambda_{\mathcal{C}}^{\mathbb{Z}}:=\mathbb{Z}\left[H_{1}(X ; \mathbb{Z})\right]$. This module is called the Alexander module of $\mathcal{C}$ and will be denoted by $M_{\mathcal{C}}^{\mathbb{Z}}$. Tensoring by a field $\mathbb{K}$ one can regard $M_{\mathcal{C}}^{\mathbb{K}}:=H_{1}(\tilde{X} ; \mathbb{K})$ as a $\Lambda_{\mathcal{C}}^{\mathbb{K}}$-module, where $\Lambda_{\mathcal{C}}^{\mathbb{K}}:=\mathbb{K}\left[H_{1}(X ; \mathbb{Z})\right]$. When refering to $\mathbb{K}=\mathbb{C}$ we may drop the superscripts.

The sequence of characteristic varieties is defined in a very natural way as a sequence of invariants of $M_{\mathcal{C}}$ by means of the reduced support of its successive exterior powers, that is,

$$
\operatorname{Char}_{k}(\mathcal{C}):=\left(\operatorname{Supp}_{\Lambda_{\mathcal{C}}}\left(\wedge^{k} M_{\mathcal{C}}\right)\right)_{\text {red }}
$$

Hence each $\operatorname{Char}_{k}(\mathcal{C})$ might be regarded as a variety in the complex torus $\operatorname{Spec}_{\mathcal{C}}=$ $\left(\mathbb{C}^{*}\right)^{r}$ whose coordinates $t_{1}, \ldots, t_{r}$ are given by the meridians of the curve $\mathcal{C}^{\prime}$-see [13] or [7] for a more detailed definition.

Characteristic varieties may also be considered over an arbitrary field $\mathbb{K},-$ cf. [16], 18]. Such varieties, defined over the torus $\left(\mathbb{K}^{*}\right)^{r}=\operatorname{Spec} \Lambda_{\mathcal{C}}^{\mathbb{K}}$, will be denoted by $\operatorname{Char}_{k}(\mathcal{C}, \mathbb{K})$.

In [1], Arapura proved, in a more general context, that $\operatorname{Char}_{k}(\mathcal{C})$ consists of a finite union of tori translated by torsion points.

We will define the terms coordinate and essential when refering to an irreducible component of $\mathrm{Char}_{k}(\mathcal{C})$.

Date: February 1, 2008.

2000 Mathematics Subject Classification. Primary 32S50, 14H30, 14Q05, 14B05, 14F45; Secondary 32Q55, 32S20, 14H10.

Key words and phrases. Characteristic varieties, fundamental group, finite coverings, sextic curves, Alexander invariants.

*Partially supported by BFM2001-1488-C02-02.

$\dagger$ Partially supported by BFM2001-1488-C02-01. 
Definition 1.1. An irreducible component $V$ of $\operatorname{Char}_{k}(\mathcal{C})$ is called a coordinate component if $V$ is contained in a coordinate torus, that is, if $V \subset \mathbb{T}_{i}:=$ $\left\{\left(t_{1}, \ldots, t_{r}\right) \in\left(\mathbb{C}^{*}\right)^{r} \mid t_{i}=1\right\}$ for some $i$.

Note that the inclusion of topological spaces $X \hookrightarrow X_{i}(i \in\{1, \ldots, r\})$, where $X_{i}:=\mathbb{P}^{2} \backslash \mathcal{C}(i)$ and $\mathcal{C}(i):=\mathcal{C}_{0} \cup \mathcal{C}_{1} \cup \ldots \cup \mathcal{C}_{i-1} \cup \mathcal{C}_{i+1} \cup \ldots \cup \mathcal{C}_{r}$, produces an injection of characteristic varieties as follows:

$$
\operatorname{Char}_{k}(\mathcal{C}(i)) \hookrightarrow \operatorname{Char}_{k}(\mathcal{C}) .
$$

Definition 1.2. An irreducible component $V$ of $\operatorname{Char}_{k}(\mathcal{C})$ is called a non-essential component if $V$ is contained in the image by (11) for some $i$, that is, if $V \subset$ $\mathrm{Char}_{k}(\mathcal{C}(i))$. Otherwise $V$ is called essential.

Remark 1.3. The concepts of essential and coordinate components depend on the embedding of $\mathcal{C}$ in $\mathbb{P}^{2}$ and not just on its complement $X$. For instance, consider the affine Ceva(2) arrangement - cf. [6] p. 80 - defined by $\mathcal{C}_{1}:=\left(x^{2}-y^{2}\right)\left(x^{2}-\right.$ $\left.z^{2}\right)\left(y^{2}-z^{2}\right) z$ and a conic, $Q=3(x y+x z+y z)+\left(x^{2}+y^{2}+z^{2}\right)$, passing through the double points of $\mathcal{C}_{1}$. Let $\mathcal{C}=\mathcal{C}_{1} \cup Q$. Figure 1.3 shows the combinatorics of the curve $\mathcal{C}$.

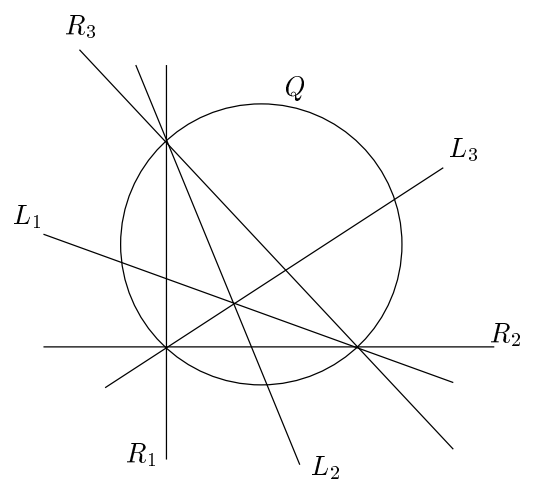

Figure 1. Curve $\mathcal{C}$.

Consider $r_{1}, r_{2}, r_{3}, \ell_{1}, \ell_{2}, \ell_{3}, \ell_{\infty}$ and $q$ meridians around the components $R_{1}, R_{2}, R_{3}, L_{1}, L_{2}, L_{3}, L_{\infty}$ and $Q$ respectively. Hence

$$
H_{1}\left(\mathbb{P}^{2} \backslash \mathcal{C} ; \mathbb{Z}\right)=\left\langle r_{i}, \ell_{j}, q: i, j \in\{1,2,3\}\right\rangle \cong \mathbb{Z}^{7}
$$

Performing a Cremona transformation based on the singular points of order four, one obtains an analytic isomorphism of the complements which induces the following isomorphism on homology.

$$
\begin{array}{cccc}
H_{1}\left(\mathbb{P}^{2} \backslash \mathcal{C} ; \mathbb{Z}\right)=\left\langle r_{i}, \ell_{j}, q\right\rangle & \stackrel{\varphi}{\mapsto} & H_{1}\left(\mathbb{P}^{2} \backslash \mathcal{C} ; \mathbb{Z}\right)=\left\langle e_{i}, \ell_{j}, \ell_{\infty}\right\rangle \\
r_{i} & \mapsto & \ell_{\infty}-\left(\ell_{i}+e_{j}+e_{k}\right) \\
\ell_{i} & \mapsto & \ell_{i} \\
q & & & \left(e_{1}+e_{2}+e_{3}\right)-\left(\ell_{1}+\ell_{2}+\ell_{3}\right),
\end{array}
$$

where $e_{i}$ is a meridian around the exceptional component $E_{i}$ resulting from blowing up the point $R_{j} \cap R_{k}(\{i, j, k\}=\{1,2,3\})$. 
One can calculate $\operatorname{Char}_{1}(\mathcal{C})$ as

$$
\begin{gathered}
\mathbb{T}=\left\{t_{q}=1\right\} \cap\left(\left\{t_{\ell_{1}} t_{\ell_{2}} t_{\ell_{3}}=t_{r_{1}}=t_{r_{2}}=t_{r_{3}}=1\right\} \cup\right. \\
\left.\bigcup_{\{i, j, k\}=\{1,2,3\}}\left\{t_{r_{i}} t_{r_{j}} t_{\ell_{k}}=t_{\ell_{i}}=t_{\ell_{j}}=t_{r_{k}}=1\right\}\right)
\end{gathered}
$$

resulting in four (non-essential) coordinate components.

Meanwhile,

$$
\begin{gathered}
\varphi(\mathbb{T})=\left\{t_{e_{1}} t_{\ell_{2}} t_{e_{3}}=t_{\ell_{1}} t_{e_{2}} t_{\ell_{3}}\right\} \cap \\
\left(\left\{t_{\ell_{1}} t_{\ell_{2}} t_{\ell_{3}}=1, t_{\infty}=t_{\ell_{i}} t_{e_{j}} t_{e_{k}}\right\}_{\{i, j, k\}=\{i, j, k\}} \cup\right. \\
\left.\bigcup_{\{i, j, k\}=\{1,2,3\}}\left\{t_{e_{i}}^{2}=t_{\infty}=t_{\ell_{i}} t_{e_{j}} t_{e_{k}}, t_{\ell_{j}}=t_{\ell_{k}}=1\right\}\right)
\end{gathered}
$$

resulting in one (essential) non-coordinate component and three (non-essential) coordinate components.

In the future we will refer to coordinate and non-coordinate components of $\operatorname{Char}_{k}(\mathcal{C})$ relative to the torus whose coordinates are meridians of the curve $\mathcal{C}^{\prime}$ in $\mathbb{C}^{2}$.

Essential non-coordinate components have been studied by Libgober in [13]. In this work, the theory of ideals of quasiadjunction was developed to fully determine such components. Also, an interpretation of the tangent cone of characteristic varieties at the identity was given by Cohen-Suciu [8] (for hyperplane arrangements) and Libgober [14] (for complements of curves).

Coordinate components of positive dimension are characterized in [13] as follows.

Lemma 1.4. Positive dimensional coordinate components of $\mathrm{Char}_{1}(\mathcal{C})$ are nonessential.

Our purpose in this note is to point out the importance of essential coordinate components. In section 2 we give a characterization for the existence of essential coordinate components. In section 3 we present a Zariski pair of curves - that is, with the same combinatorics but non-homeomorphic complements, cf. [2]that can be distinguished only by essential coordinate components of their first characteristic varieties - examples of essential coordinate components in the second characteristic varieties were given by Cohen and Suciu in [8]. In section 1 t we correlate essential coordinate components to ideals of quasiadjuntion. Finally, in section 5 we give a topological interpretation of such components.

\section{CoOrdinate And NON-ESSEntial COMPOnEnts}

For simplicity we will use the following notation:

(1) $X_{i}:=\mathbb{P}^{2} \backslash \mathcal{C}(i)$, where $\mathcal{C}(i):=\mathcal{C}_{0} \cup \cdots \cup \mathcal{C}_{i-1} \cup \mathcal{C}_{i+1} \cup \cdots \cup \mathcal{C}_{r}$.

(2) $\Lambda^{\bullet}:=\Lambda_{\mathcal{C}}^{\bullet}, M^{\bullet}:=M_{\mathcal{C}}^{\bullet}, \Lambda_{i}^{\bullet}:=\Lambda_{\mathcal{C}(i)}^{\bullet}$ and $M_{i}^{\bullet}:=M_{\mathcal{C}(i)}^{\bullet}$, where $\bullet=\mathbb{Z}$ or $\mathbb{K}$. 
(3) Note that given a $\operatorname{ring} A$ and an $A$-module $M$, we may define

$$
\operatorname{Char}_{A, k}(M):=\left(\operatorname{Supp}_{A}\left(\wedge^{k} M\right)\right)_{\mathrm{red}} \text {. }
$$

If $A \stackrel{\varphi}{\rightarrow} B$ is a morphism of rings and $M$ is a $B$-module, then there is a morphism $\operatorname{Char}_{A, k}(M) \stackrel{\varphi^{*}}{\rightarrow} \operatorname{Char}_{B, k}(M)$. In our case we will regard $\operatorname{Char}_{\Lambda_{i}^{\mathbb{K}}, k}\left(M_{i}^{\mathbb{K}}\right)$ as a subvariety of $\operatorname{Spec} \Lambda^{\mathbb{K}}$ via the inclusion $\operatorname{Spec} \Lambda_{i}^{\mathbb{K}} \hookrightarrow \operatorname{Spec} \Lambda^{\mathbb{K}}=\left(\mathbb{K}^{*}\right)^{r}$, which identifies $\operatorname{Spec} \Lambda_{i}^{\mathbb{K}}$ and $\mathbb{T}_{i}^{\mathbb{K}}:=\left\{\left(t_{1}, \ldots, t_{r}\right) \in\left(\mathbb{K}^{*}\right)^{r} \mid t_{i}=1\right\}$. Such a variety, contained in $\mathbb{T}_{i}^{\mathbb{K}}$, will always be denoted by $\operatorname{Char}_{k}\left(\mathcal{C}_{i}, \mathbb{K}\right)$.

An alternative way to calculate the characteristic varieties of a module is via the Fitting ideals of a free resolution - cf. [12], [15]. Let

$$
\left(\Lambda^{\mathbb{K}}\right)^{m} \stackrel{\phi}{\rightarrow}\left(\Lambda^{\mathbb{K}}\right)^{n} \rightarrow M^{\mathbb{K}} \rightarrow 0
$$

be a free resolution of $M^{\mathbb{K}}$. The map $\phi$ defines a $n \times m$ matrix with coefficients in $\Lambda^{\mathbb{K}}$. The Fitting ideal $F_{k}\left(M^{\mathbb{K}}\right)$ of $M^{\mathbb{K}}$ is defined as the ideal generated by:

$$
\begin{cases}0 & \text { if } k \leq \max \{0, n-m\} \\ 1 & \text { if } k>n \\ \text { minors of } \phi \text { of order }(n-k+1) & \text { otherwise. }\end{cases}
$$

One can therefore define

$$
V_{k}(\mathcal{C}, \mathbb{K})=V_{k}\left(M^{\mathbb{K}}\right):=\operatorname{Supp}_{\Lambda^{\mathbb{K}}}\left(M^{\mathbb{K}} / F_{k}\left(M^{\mathbb{K}}\right)\right) .
$$

An interesting remark is that $\left(V_{k}(\mathcal{C}, \mathbb{K})\right)_{\text {red }}=\operatorname{Char}_{k}(\mathcal{C}, \mathbb{K})(\operatorname{cf} .[13$, 15]).

Suppose one has a finite presentation of $\pi_{1}(X)$ whose generators are meridians of the components of $\mathcal{C}^{\prime} \subset \mathbb{C}^{2}$. This allows one to obtain a finite free resolution of the relative homology group $M^{\prime}:=H_{1}(\tilde{X}, \tilde{p} ; \mathbb{Z})$ via Fox Calculus, where $\tilde{p}$ denotes the inverse image of a point $p \in X$ by the universal abelian cover. Such a finite free resolution has the form

$$
\left(\Lambda^{\mathbb{K}}\right)^{m} \stackrel{\phi}{\rightarrow}\left(\Lambda^{\mathbb{K}}\right)^{n} \rightarrow M^{\prime} \otimes \mathbb{K} \rightarrow 0
$$

where $m$ is the number of relations and $n$ the number of generators of the given presentation of $\pi_{1}(X)$. The widely used connection between $\operatorname{Char}_{k}(\mathcal{C}, \mathbb{K})$ and $\operatorname{Char}_{\Lambda^{\mathbb{K}}, k}\left(M^{\prime} \otimes \mathbb{K}\right)$ reads as follows:

$$
\operatorname{Char}_{k}^{*}(\mathcal{C}, \mathbb{K})=\operatorname{Char}_{\Lambda^{\mathbb{K}}, k}^{*}\left(M^{\prime} \otimes \mathbb{K}\right)
$$

where $\operatorname{Char}_{k}^{*}$ denotes $\operatorname{Char}_{k}$ minus the origin $\overline{1}=(1, \ldots, 1) \in\left(\mathbb{K}^{*}\right)^{r}$, see for instance [7].

Consider the morphism $\sigma_{i}$ induced by the inclusion:

$$
G:=\pi_{1}(X) \stackrel{\sigma_{i}}{\rightarrow} G_{i}:=\pi_{1}\left(X_{i}\right) .
$$

This morphism is a surjection whose kernel is normally generated by $\gamma_{i}$, a meridian of $\mathcal{C}_{i}$. from the presentation of $G$ one can construct a free resolution of $M_{i}^{\prime}$

$$
\left(\Lambda_{i}^{\mathbb{Z}}\right)^{m+1} \stackrel{\phi_{i}}{\rightarrow}\left(\Lambda_{i}^{\mathbb{Z}}\right)^{n} \rightarrow M_{i}^{\prime} \rightarrow 0
$$


by adding the relation $\gamma_{i}=1$. Hence, the matrix $\phi_{i}$ is obtained from $\phi$ by evaluating on $t_{i}=1$ and adding a column with zeroes everywhere except in the position corresponding to $\gamma_{i}$, where a 1 is located:

$$
\phi_{i}=\left(\begin{array}{cc} 
& 0 \\
& \vdots \\
& 0 \\
& 1 \\
& 0 \\
& \vdots \\
& 0
\end{array}\right) .
$$

Therefore one has the following:

Lemma 2.1. Under the previous conditions

$$
\operatorname{Char}_{k}^{*}\left(\mathcal{C}_{i}, \mathbb{K}\right) \subset \operatorname{Char}_{k}^{*}(\mathcal{C}, \mathbb{K}) \cap \mathbb{T}_{i}^{\mathbb{K}}
$$

Proof. Using (3) it is easy to check the following inclusion of ideals

$$
F_{k+1}\left(M_{i}^{\prime}\right)+\left(t_{i}-1\right) \supset F_{k+1}\left(M^{\prime}\right)+F_{k+2}\left(M^{\prime}\right)+\left(t_{i}-1\right) .
$$

Hence, since $F_{k+2}\left(M^{\prime}\right) \subset F_{k+1}\left(M^{\prime}\right)$, the result follows.

The inequality can be strict. This is illustrated by Cohen-Suciu in [8, Example 4.4], for $k=2$, using an arrangement of lines in $\mathbb{P}^{2}$. In this work we present an example of strict inequality for $k=1$. Note that, by Lemma 1.4, the difference consists only of a finite union of points.

The variety $\operatorname{Char}_{k}(\mathcal{C}, \mathbb{K}) \cap \mathbb{T}_{i}^{\mathbb{K}}$ has the following interpretation.

\section{Lemma 2.2.}

$$
\operatorname{Char}_{k}(\mathcal{C}, \mathbb{K}) \cap \mathbb{T}_{i}^{\mathbb{K}}=\operatorname{Char}_{\Lambda^{\mathbb{K}}, k}\left(M(i)^{\mathbb{K}}\right),
$$

where $M(i)^{\mathbb{K}}:=M \otimes\left(\Lambda^{\mathbb{K}} /\left(t_{i}-1\right)\right)$.

Proof. Tensoring a free resolution of $M^{\mathbb{Z}}$ by $\Lambda^{\mathbb{K}} /\left(t_{i}-1\right)$ produces a free resolution of $M(i)^{\mathbb{K}}$ as a $\Lambda_{i}^{\mathbb{K}}$-module. Hence, $F_{k}\left(M(i)^{\mathbb{K}}\right)$ can be identified with the ideal $\operatorname{sum} F_{k}\left(M^{\mathbb{K}}\right)+\left(t_{i}-1\right)$.

Note that $M(i)^{\mathbb{Z}}$ is related to a certain cover of $X$, say $\tilde{X}(i)$, that fits in the following diagram

$$
\begin{aligned}
& \tilde{X} \stackrel{t_{i}}{\longrightarrow} \tilde{X}(i) \\
& \downarrow \rho \swarrow \rho_{i} \\
& X
\end{aligned}
$$

where $\rho$ is the universal abelian cover of $X, X(i)$ is the maximal abelian cover of $X$ not ramified on $\mathcal{C}_{i}$, and $t_{i}$ is the infinite cyclic cover of $\tilde{X}(i)$ ramified on $\rho_{i}^{-1}(\mathcal{C}(i))$.

The $\Lambda_{i}^{\mathbb{Z}}$-modules $M(i)^{\mathbb{Z}}$ and $H_{1}(\tilde{X}(i) ; \mathbb{Z})$ fit in the following short exact sequence

$$
0 \rightarrow M(i)^{\mathbb{Z}} \rightarrow H_{1}(\tilde{X}(i) ; \mathbb{Z}) \rightarrow \Lambda_{i}^{\mathbb{Z}} / I_{i}=\mathbb{Z} \rightarrow 0,
$$

where $I_{i}$ is the augmentation ideal of $\Lambda_{i}^{\mathbb{Z}}$. 
Notation 2.3. If $\varphi$ denotes an unbranched cover of a path-connected topological space $Z$, say $Y \stackrel{\varphi}{\rightarrow} Z$, the same notation will be kept for the inclusion of subgroups $\pi_{1}(Y) \stackrel{\varphi}{\hookrightarrow} \pi_{1}(Z)$.

As a subgroup of $G$, the cover $\tilde{X}(i)$ corresponds to the smallest normal subgroup $K \triangleleft G$ of $G$ containing $\gamma_{i}$ (a meridian of $\mathcal{C}_{i}$ ) such that $G / K$ is abelian. Therefore $K=\sigma_{i}^{-1}\left(G_{i}^{\prime}\right)$, where $G_{i}^{\prime}$ denotes the commutator subgroup of $G_{i}$. Hence one has the following exact sequence of $\Lambda_{i}^{\mathbb{Z}}$-modules

$$
0 \rightarrow \tilde{R}(i)^{\mathbb{Z}} \rightarrow H_{1}(\tilde{X}(i) ; \mathbb{Z}) \rightarrow H_{1}\left(\tilde{X}_{i} ; \mathbb{Z}\right) \rightarrow 0
$$

where

$$
\tilde{R}(i)^{\mathbb{Z}}=\frac{\sigma_{i}^{-1}\left(G_{i}^{\prime \prime}\right)}{\left[\sigma_{i}^{-1}\left(G_{i}^{\prime}\right), \sigma_{i}^{-1}\left(G_{i}^{\prime}\right)\right]} .
$$

Note that the morphism $M(i)^{\mathbb{Z}} \rightarrow H_{1}\left(\tilde{X}_{i} ; \mathbb{Z}\right)$ is surjective.

Definition 2.4. Let us denote by $R(i)^{\bullet}$ the kernel of the natural epimorphism

$$
0 \rightarrow R(i)^{\bullet} \rightarrow M(i)^{\bullet} \rightarrow H_{1}\left(\tilde{X}_{i} ; \bullet\right) \rightarrow 0
$$

obtained from (1).

Such a $\Lambda_{i}^{\mathbb{Z}}$-module will be called the residual module of $X$ with respect to $\mathcal{C}_{i}$ (with coefficients in $\bullet=\mathbb{Z}, \mathbb{K}$ ).

From (4) and (5) it is obvious that $R(i)^{\mathbb{Z}}$ can also be obtained as the intersection of $\tilde{R}(i)^{\mathbb{Z}}$ and $M(i)^{\mathbb{Z}}$, that is, as the kernel of

$$
\tilde{R}(i)^{\mathbb{Z}} \rightarrow \Lambda_{i}^{\mathbb{Z}} / I_{i}
$$

The residual module measures the existence of non-essential coordinate components.

Corollary 2.5. The reduced residual module $R^{\mathbb{K}}(i)_{\mathrm{red}}$ is trivial if and only if $\operatorname{Char}_{1}(\mathcal{C}, \mathbb{K}) \cap \mathbb{T}_{i}^{\mathbb{K}}$ consists of non-essential components.

Proof. The result is a consequence of Lemma 2.2 and the following remarks:

(1) $M(i)^{\mathbb{K}}=H_{1}(\tilde{X} ; \mathbb{Z}) \otimes \Lambda^{\mathbb{K}} /\left(t_{i}-1\right)$

(2) $M_{i}^{\mathbb{Z}}=H_{1}\left(\tilde{X}_{i} ; \mathbb{Z}\right)$

Remark 2.6. Recall that non-coordinate essential components may be computed by means of Libgober's method [13]. Then, the knowledge of non-coordinate essential components and $R^{\mathbb{K}}(i)_{\text {red }}, i=1, \ldots, r$ characterize essential coordinate components of $\operatorname{Char}_{1}(\mathcal{C}, \mathbb{K})$. 


\section{A ZARISKI PAIR}

Consider the space $\mathcal{M}$ of sextics with the following combinatorics:

(1) $\mathcal{C}$ is a union of a smooth conic $\mathcal{C}_{2}$ and a quartic $\mathcal{C}_{4}$.

(2) $\operatorname{Sing}\left(\mathcal{C}_{4}\right)=\{P, Q\}$ where $Q$ is a cusp of type $\mathbb{A}_{4}$ and $P$ is a node of type $\mathbb{A}_{1}$.

(3) $\mathcal{C}_{2} \cap \mathcal{C}_{4}=\{Q, R\}$ where $Q$ is a $\mathbb{D}_{7}$ on $\mathcal{C}$ and $R$ is a $\mathbb{A}_{11}$ on $\mathcal{C}$.

Performing a degenerated Cremona transformation based on $2 Q$ and $R$, the problem is equivalent to finding a nodal cubic $\tilde{\mathcal{C}}_{4}$ and a smooth conic $\tilde{\mathcal{C}}_{2}$ intersecting in two singular points of types $\mathbb{A}_{9}$ and $\mathbb{A}_{1}$.
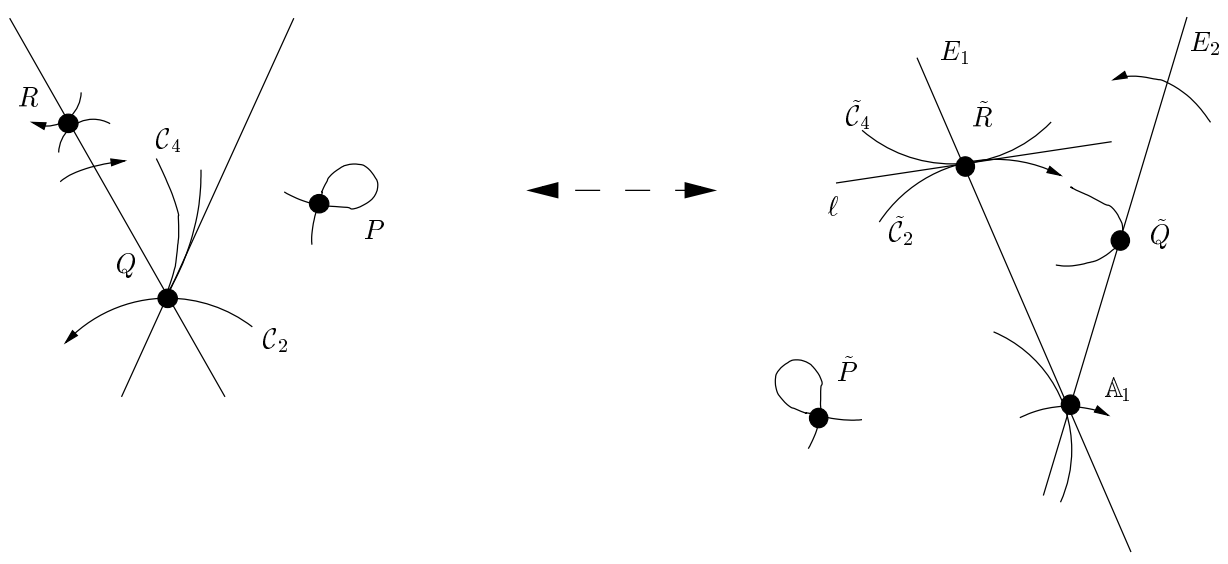

FiguRE 2. Cremona transformation.

Assuming $\tilde{\mathcal{C}_{4}}$ has equation $x y z+x^{3}-y^{3}$, one can consider the following parametrization

$$
\begin{array}{rlc}
\varphi: \mathbb{C} & \rightarrow & \tilde{\mathcal{C}}_{4} \\
t & \mapsto & {\left[t: t^{2}: t^{3}-1\right] .}
\end{array}
$$

Note that $\left.\varphi\right|_{\mathbb{C}^{*}}: \mathbb{C}^{*} \rightarrow \operatorname{Reg}\left(\tilde{\mathcal{C}}_{4}\right)$ is a group isomorphism from the multiplicative group $\mathbb{C}^{*}$ to the set of regular points on the cubic $\tilde{\mathcal{C}}_{4}$ whose geometric group structure has the inflexion point $\varphi(1)=[1: 1: 0]$ as unity.

Let $t_{1}, t_{2}$ and $t_{3}$ denote the parameters corresponding to $\tilde{R}, \mathbb{A}_{1}$ and $\tilde{Q}$ respectively. One has the following relations given by $E_{1}, \tilde{\mathcal{C}}_{2}$ and $E_{2}$ :

$$
\begin{gathered}
t_{1} t_{2}^{2}=1 \\
t_{1}^{5} t_{2}=1 \\
t_{2} t_{3}^{2}=1 .
\end{gathered}
$$

This implies that $t_{1}$ is a ninth root of 1 , say $\alpha$, and $t_{2}=\alpha^{4}$. Therefore $\alpha$ must also be a primitive ninth root of unity. That leaves us with two possibilities for $t_{3}$, namely, $t_{3}= \pm \alpha^{7}$. The solution $-\alpha^{7}$ (resp. $+\alpha^{7}$ ) corresponds to the case where the tangent line to $\tilde{\mathcal{C}}_{4}$ at $\tilde{R}$ passes (resp. doesn't pass) through $\tilde{Q}$. 
Using the techniques shown in [3], one can obtain equations for two sextics $\mathcal{C}_{6}^{(1)}=\mathcal{C}_{4}^{(1)} \cup \mathcal{C}_{2}^{(1)}$ and $\mathcal{C}_{6}^{(2)}=\mathcal{C}_{4}^{(2)} \cup \mathcal{C}_{2}^{(2)}$ satisfying the properties stated above and an extra property: there exists a conic $\tilde{\ell}$-the inverse image of $\ell$ - passing through $R$ and $Q$ such that $\operatorname{mult}_{R}\left(\tilde{\ell}, \mathcal{C}_{2}^{(i)}\right)=\operatorname{mult}_{R}\left(\tilde{\ell}, \mathcal{C}_{4}^{(i)}\right)=3, \operatorname{mult}_{Q}\left(\tilde{\ell}, \mathcal{C}_{2}^{(i)}\right)=1$ and $\operatorname{mult}_{Q}\left(\tilde{\ell}, \mathcal{C}_{4}^{(i)}\right)=3+i$.

Note that, by construction, $\mathcal{C}_{6}^{(1)}$ and $\mathcal{C}_{6}^{(2)}$ belong to different components of $\mathcal{M}$. Moreover, if we consider the action of $\operatorname{PGL}(3, \mathbb{C})$ on $\mathcal{M}$, then $\mathcal{M} / P G L(3, \mathbb{C})$ consists of exactly two points having representatives $\mathcal{C}_{6}^{(1)}$ and $\mathcal{C}_{6}^{(2)}$.

Special affine equations for these curves are shown below. The affine coordinates are $(y, z)$; the line at infinity is tangent to the type $\mathbb{D}_{7}$ point, which is the base point of the pencil of vertical lines $y=$ constant:

$$
f_{1}(y, z):=\left((y+3) z+\frac{3 y^{2}}{2}\right)\left(z^{2}-\left(y^{2}+\frac{15}{2} y+\frac{9}{2}\right) z-3 y^{3}-\frac{9 y^{2}}{4}+\frac{y^{4}}{4}\right)
$$

for $\mathcal{C}_{6}^{(1)}$ and

$$
f_{2}(y, z):=\left(\left(y+\frac{1}{3}\right) z-\frac{y^{2}}{6}\right)\left(z^{2}-\left(y^{2}+\frac{9 y}{2}+\frac{3}{2}\right) z+\frac{y^{4}}{4}+\frac{3 y^{2}}{4}\right)
$$

for $\mathcal{C}_{6}^{(2)}$.

In the future we will refer to $\mathcal{C}^{(i)} i=1,2$ as the union of the sextic curve $\mathcal{C}_{6}^{(i)}$ and a transversal line $\mathcal{C}_{0}$, where $\mathbb{C}^{2}=\mathbb{P}^{2} \backslash \mathcal{C}_{0}$ and $X^{(i)}=\mathbb{P}^{2} \backslash \mathcal{C}^{(i)}$.

Proposition 3.1. The fundamental groups $G^{(i)}:=\pi_{1}\left(X^{(i)}\right)$ have the following presentations

$$
\begin{gathered}
G^{(1)}=\left\langle e_{1}, e_{2}:\left[e_{2}, e_{1}^{2}\right]=1,\left(e_{1} e_{2}\right)^{2}=\left(e_{2} e_{1}\right)^{2},\left[e_{1}, e_{2}^{2}\right]=1\right\rangle \\
G^{(2)}=\left\langle e_{1}, e_{2}:\left[e_{2}, e_{1}^{2}\right]=1,\left(e_{1} e_{2}\right)^{2}=\left(e_{2} e_{1}\right)^{2}\right\rangle .
\end{gathered}
$$

Sketch of the proof. The main ideas of this proof have already appeared in [5]. Let us begin by considering the affine curves $\hat{\mathcal{C}}_{6}^{(i)}$ defined by $f_{i}(y, z)=0$ and the projection $(y, z) \mapsto y$. Note that $\mathbb{C}^{2} \backslash \tilde{\mathcal{C}}_{6}^{(i)}$ cannot be $X^{(i)}$ because of the non-generic choice of the line at infinity.

Step 1. Computation of the braid monodromy of $\hat{\mathcal{C}}_{6}^{(i)}$.

We follow the method introduced in [5] for "curves with real pictures" to obtain a braid monodromy. In both cases, all the roots of the discriminant $\Delta$ of $f_{i}(y, z)$ with respect to $z$ are real.

Figure 3 shows the real pictures of $\hat{\mathcal{C}}_{6}^{(i)}$. The dashed lines correspond to the nontransversal vertical lines; the dotted stretches represent the real parts of complex conjugate solutions and the thickened points represent real nodes with imaginary tangent lines. The thickened curves represent the conics.

Braid monodromy is computed for closed paths as follows. Let us choose a big real number $*_{y}$ as the base point. We order the points in the discriminant $y_{1}>\cdots>y_{4}$ and fix $\varepsilon>0$ small enough. We use the following notation: 


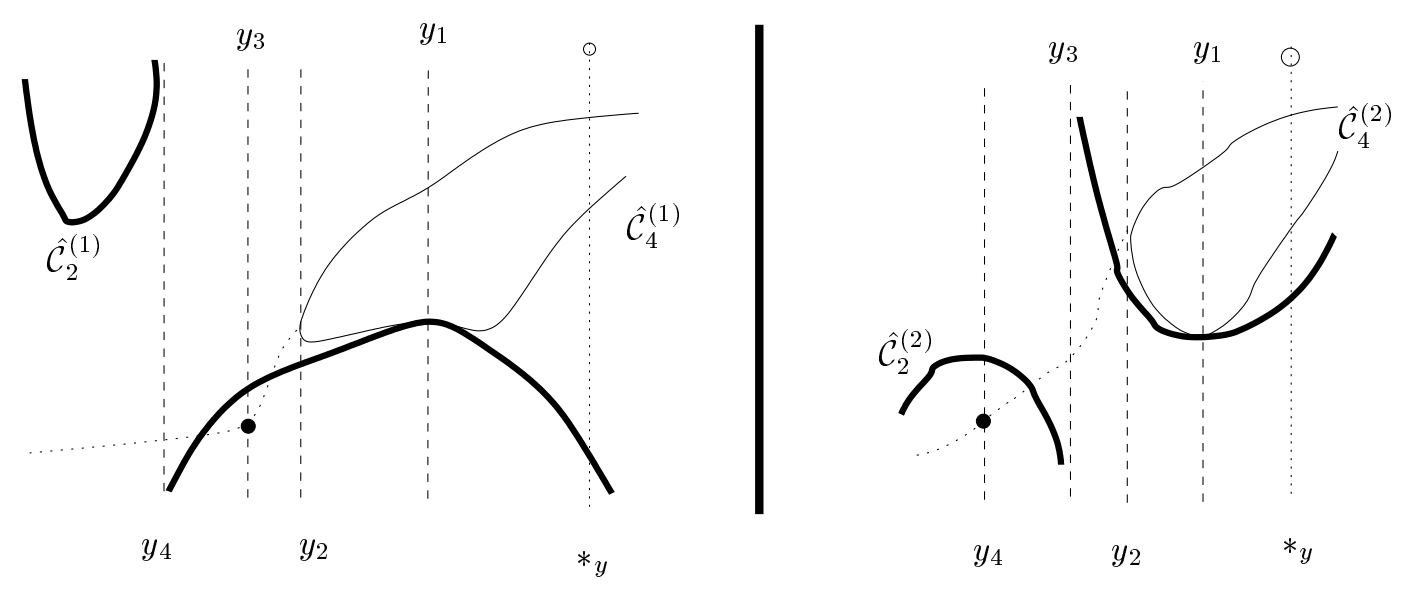

FiguRE 3. The left figure is $\hat{\mathcal{C}}_{6}^{(1)}$ and the right figure is $\hat{\mathcal{C}}_{6}^{(2)}$.

- $\gamma_{1}$ for the segment in the real line from $*_{y}$ to $y_{1}+\varepsilon$.

- $\gamma_{j}, j=2,3,4$, for the segment in the real line from $y_{j-1}-\varepsilon$ to $y_{j+1}+\varepsilon$.

- $\delta_{j}, j=1, \ldots, r$ for the closed path based at $y_{j}+\varepsilon$, which runs counterclockwise along the circle of radius $\varepsilon$ centered at $y_{j}$; the upper half is denoted by $\delta_{j}^{+}$and the lower half is denoted by $\delta_{j}^{-}$.

Braid monodromy is computed along the paths

$$
\eta_{j}:=\gamma_{1} \cdot\left(\prod_{k=2}^{j}\left(\gamma_{k} \cdot \delta_{j}^{+}\right)\right) \cdot \delta_{j} \cdot\left(\gamma_{1}\left(\prod_{k=2}^{j}\left(\gamma_{k} \cdot \delta_{j}^{+}\right)\right)\right)^{-1},
$$

$j=1, \ldots, 4$. We follow the method and conventions for "curves with real pictures" introduced in [5] to obtain the braid monodromies, (see Table 1 and 2).

\begin{tabular}{|c|c|c|c|}
\hline$\eta_{1}$ & $\eta_{2}$ & $\eta_{3}$ & $\eta_{4}$ \\
\hline$\sigma_{2}^{12}$ & $\sigma_{2}^{6} * \sigma_{1}$ & $\sigma_{2}^{4} * \sigma_{1}^{2}$ & $\left(\sigma_{2}^{4} \sigma_{1} \sigma_{2}^{2}\right) *\left(\sigma_{2} \sigma_{1}^{2} \sigma_{2}\right)^{-1}$ \\
\hline
\end{tabular}

TABLE 1. Braid monodromy for $\hat{\mathcal{C}}_{6}^{(1)}$

\begin{tabular}{|c|c|c|c|}
\hline$\eta_{1}$ & $\eta_{2}$ & $\eta_{3}$ & $\eta_{4}$ \\
\hline$\sigma_{2}^{12}$ & $\sigma_{2}^{6} * \sigma_{1}$ & $\left(\sigma_{2}^{5} \sigma_{1}\right) *\left(\sigma_{1} \sigma_{2}^{2} \sigma_{1}\right)^{-1}$ & $\sigma_{2}^{2} * \sigma_{1}^{2}$ \\
\hline
\end{tabular}

TABLE 2. Braid monodromy for $\hat{\mathcal{C}}_{6}^{(2)}$

The notation $\sigma * \tau$ represents $\sigma \tau \sigma^{-1}$.

Step 2. Computation of the fundamental group of $\hat{\mathcal{C}}_{6}^{(i)}$. 
Note that the standard Zariski-van Kampen method cannot be applied in this instance due to vertical asymptotes. A generalized Zariski-van Kampen method applicable to this case can be found in 4 .

Let us choose a basis $a_{1}, a_{2}, a_{3}$ of the fundamental group of the fiber on $*_{y}$ as in the case of the fundamental group of the complement of the discriminant. These elements also generate $\pi_{1}\left(\mathbb{C}^{2} \backslash \hat{\mathcal{C}}_{6}^{(i)}\right)$. The standard action of the braid group on the free group generated by $a_{1}, a_{2}, a_{3}$ is defined as

$$
a_{j}^{\sigma_{i}}= \begin{cases}a_{j} & \text { if } j \neq i, i+1 \\ a_{j+1} & \text { if } j=i \\ a_{j+1} * a_{i} & \text { if } j=i+1 .\end{cases}
$$

Let us denote by $\tau_{i}$ the image of $\eta_{i}$ by the braid monodromy. If $\eta_{j}$ does not correspond to an asymptote, the relations $a_{j}=a_{j}^{\tau_{i}}, j=1,2,3$ are satisfied. Otherwise the relations $a_{j}^{b_{i}}=a_{j}^{\tau_{i}}, j=1,2,3$ are satisfied, where

$$
b_{i}= \begin{cases}a_{3}^{a_{2} a_{3} a_{2} a_{1}} & \text { if } i=4 \text { for } \tilde{\mathcal{C}}_{6}^{(1)} \\ a_{3}^{a_{2} a_{3} a_{2} a_{3} a_{2} a_{1}} & \text { if } i=3 \text { for } \tilde{\mathcal{C}}_{6}^{(2)} .\end{cases}
$$

The generalized Zariski-van Kampen method asserts that the above relations are sufficient to give a presentation of $\pi_{1}\left(\mathbb{C}^{2} \backslash \hat{\mathcal{C}}_{6}^{(i)}\right)$.

Step 3. Computation of the fundamental group of $\mathcal{C}_{6}^{(i)}$.

In order to obtain $\pi_{1}\left(\mathbb{P}^{2} \backslash \mathcal{C}_{6}^{(i)}\right)$, it is enough to factor $\pi_{1}\left(\mathbb{C}^{2} \backslash \hat{\mathcal{C}}_{6}^{(i)}\right)$ by a meridian of the line at infinity. Blowing up the projection point one can see the fibration used for the braid monodromy as the restriction of the standard ruled fibration $\mathbb{F}_{1} \rightarrow \mathbb{P}^{1}$.

Figure 4 gives the real picture of $\hat{\mathcal{C}}_{6}^{(i)}$ around both the exceptional divisor (the horizontal line) and the strict transform of the line at infinity (the vertical line). Since the self-intersection number of the exceptional curve equals -1 , it is easily seen that $\left(b_{j} a_{2} a_{1} a_{3} a_{2} a_{1}\right)^{-1}$ is a meridian of the line at infinity.

We apply GAP4 [9] to perform Tietze transformations in order to obtain the following presentations of the fundamental groups:

$$
\begin{gathered}
\pi_{1}\left(\mathcal{C}_{6}^{(1)}\right)=\left\langle a_{1}, a_{3}:\left[a_{3}, a_{1}^{2}\right]=1,\left(a_{1} a_{3}\right)^{2}=\left(a_{3} a_{1}\right)^{2}, a_{3} a_{1} a_{3} a_{1}^{3}=1\right\rangle, \\
\pi_{1}\left(\mathcal{C}_{6}^{(2)}\right)=\left\langle a_{1}, a_{3}:\left[a_{3}, a_{1}^{2}\right]=1,\left(a_{1} a_{3}\right)^{2}=\left(a_{3} a_{1}\right)^{2},\left(a_{3} a_{1}^{2}\right)^{2}=1\right\rangle .
\end{gathered}
$$

Step 4. Computation of the fundamental group of $\mathcal{C}_{6}^{(i)}$.

We apply last step and [5, Prop. 2] to obtain the presentations of Proposition 3.1.

One can easily calculate the matrix of the free resolution of $M^{\prime(i)}$ by means of Fox Calculus to get

$$
\phi^{(2)}=\left[\begin{array}{cc}
\left(t_{1}-1\right)\left(t_{1}+1\right) & \left(t_{1}-1\right)\left(t_{1} t_{2}+1\right) \\
\left(t_{2}-1\right)\left(t_{1}+1\right) & \left(t_{2}-1\right)\left(t_{1} t_{2}+1\right)
\end{array}\right]
$$




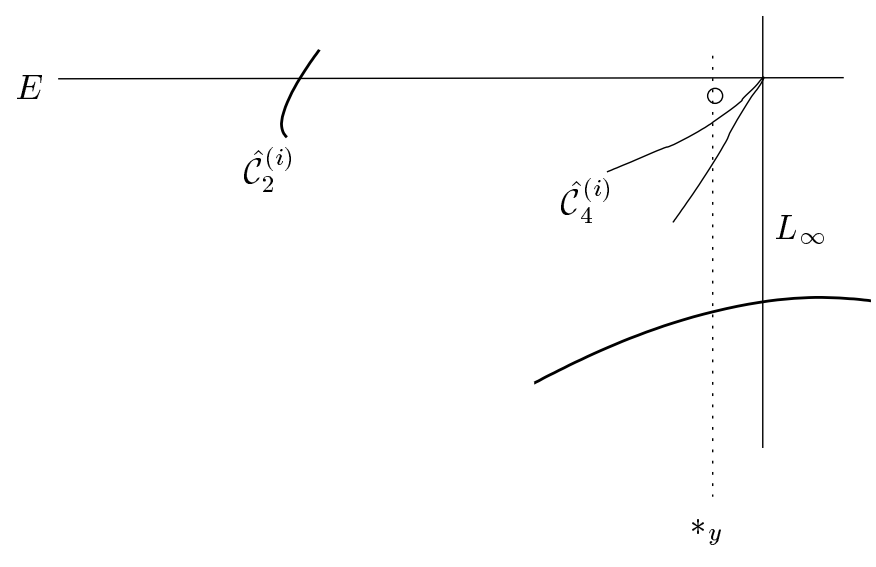

Figure 4.

and

Hence

$$
\phi^{(1)}=\left[\begin{array}{lll}
\left(t_{1}-1\right)\left(t_{1}+1\right) & \left(t_{1}-1\right)\left(t_{1} t_{2}+1\right) & \left(t_{1}-1\right)\left(t_{2}+1\right) \\
\left(t_{2}-1\right)\left(t_{1}+1\right) & \left(t_{2}-1\right)\left(t_{1} t_{2}+1\right) & \left(t_{2}-1\right)\left(t_{2}+1\right)
\end{array}\right] .
$$

$\operatorname{Char}_{1}^{*}\left(\mathcal{C}^{(2)}, \mathbb{K}\right)=\left\{\begin{array}{cl}\emptyset & \text { if } \operatorname{char}_{\mathbb{K}}=2 \\ (-1,1) & \text { otherwise }\end{array}\right.$ and $\operatorname{Char}_{1}^{*}\left(\mathcal{C}^{(1)}, \mathbb{K}\right)=\emptyset$

Using Reidemeister-Schreier's method one can explicitly obtain the short exact sequences described in (5) for both meridians $e_{1}$ and $e_{2}$ :

(1) For the coordinate components on $t_{1}=1$ :

$$
0 \rightarrow \tilde{R}^{(i) \mathbb{Z}}(1) \rightarrow \mathbb{Z} e_{1} \oplus \mathbb{Z} / 2 \mathbb{Z}\left[e_{1}, e_{2}\right] \rightarrow 0 \rightarrow 0
$$

From (6) one has $e_{1} \mapsto e_{1}$ and $\left[e_{1}, e_{2}\right] \mapsto\left(t_{2}-1\right) e_{1}$. This implies $R^{(1) \mathbb{Z}}(1)=R^{(2) \mathbb{Z}}(1)=\mathbb{Z} / 2 \mathbb{Z}\left[e_{1}, e_{2}\right]$, and therefore

$$
R^{(1) \mathbb{K}}(1)=R^{(2) \mathbb{K}}(1)= \begin{cases}\mathbb{K} & \text { if } \text { char }_{\mathbb{K}}=2 \\ 0 & \text { otherwise. }\end{cases}
$$

(2) For the coordinate components on $t_{2}=1$ :

$$
\begin{aligned}
& 0 \rightarrow \tilde{R}^{(1) \mathbb{Z}}(2) \rightarrow \mathbb{Z} e_{2} \oplus \mathbb{Z} / 2 \mathbb{Z}\left[e_{1}, e_{2}\right] \rightarrow 0 \rightarrow 0 \\
& 0 \rightarrow \tilde{R}^{(2) \mathbb{Z}}(2) \rightarrow \mathbb{Z} e_{2} \oplus \mathbb{Z}\left[e_{1}, e_{2}\right] \quad \rightarrow \quad \rightarrow \quad \rightarrow 0
\end{aligned}
$$

From (6) one has $e_{2} \mapsto e_{2}$ and $\left[e_{1}, e_{2}\right] \mapsto\left(t_{1}-1\right) e_{2}$. This implies $R^{(1) \mathbb{Z}}(2)=\mathbb{Z} / 2 \mathbb{Z}\left[e_{1}, e_{2}\right]$ and $R^{(2) \mathbb{Z}}(2)=\mathbb{Z}\left[e_{1}, e_{2}\right]$. Therefore

$$
R^{(1) \mathbb{K}}(2)=\left\{\begin{array}{ll}
\mathbb{K} & \text { if } \operatorname{char}_{\mathbb{K}}=2 \\
0 & \text { otherwise }
\end{array} \text { and } \quad R^{(2) \mathbb{K}}(2)=\mathbb{K} .\right.
$$

\footnotetext{
${ }^{1}$ For computationally more difficult problems, the authors have developed a software package programmed for Singular - 11], [10] - which is available upon request.
} 


\section{IDEALS OF QUASIADJUNCTION}

Global ideals of quasiadjunction - cf. [13] - give the required topological information to calculate the first Betti number of abelian covers of $\mathbb{P}^{2}$ ramified along a curve $\mathcal{C}$. This is based on Sakuma's formula - cf. Theorem 7.3. in [17] - for the first Betti number of abelian covers of compact smooth complex surfaces ramified along complex curves. In our case it can be described as follows. Let the manifold $\bar{X}_{\rho}$ denote a certain abelian cover of $\mathbb{P}^{2}$ ramified along $\mathcal{C}$ and let $X_{\rho}$ be its associated unbranched covering. Consider the set $\mathbb{T}_{X_{\rho}}$ of representations $H_{1}\left(X_{\rho} ; \mathbb{Z}\right) \rightarrow \mathbb{C}^{*}$ and $\mathbb{T}_{X_{\rho}}^{*}:=\mathbb{T}_{X_{\rho}} \backslash\{1\}$. Then

$$
b_{1}\left(\bar{X}_{\rho}\right)=\sum_{\xi \in \mathbb{T}_{X \rho}^{*}} \operatorname{Null}\left(\mathcal{C}_{\xi} ; \xi\right),
$$

where

$$
\operatorname{Null}\left(\mathcal{C}_{\xi} ; \xi\right):=\max \left\{k \in \mathbb{N} \mid \xi \in \operatorname{Char}_{k}\left(\mathcal{C}_{\xi}\right)\right\}
$$

and $\operatorname{Char}_{k}\left(\mathcal{C}_{\xi}\right) \subset \mathbb{T}^{\xi}$ which is the coordinate subtorus of $\mathbb{T}_{X_{\rho}}$ determined by the trivial coordinates of $\xi$. The curve $\mathcal{C}_{\xi}$ denotes the union of components of $\mathcal{C}$ associated with the non-trivial coordinates of $\xi$. Libgober proved that $\operatorname{Null}\left(\mathcal{C}_{\xi} ; \xi\right)$ coincides with the irregularity of a certain ideal sheaf on $\mathbb{P}^{2}$ associated with $\xi$ and $\mathcal{C}$.

Alternatively, Sakuma also provided the following formula for the first Betti number of $X_{\rho}$ :

$$
b_{1}\left(X_{\rho}\right)=b_{1}(X)+\sum_{\xi \in \mathbb{T}_{X \rho}^{*}} \operatorname{Null}(\mathcal{C} ; \xi) .
$$

Remark 4.1. Note that $\operatorname{Null}(\mathcal{C} ; \xi)=\operatorname{Null}\left(\mathcal{C}_{\xi} ; \xi\right)$ if no coordinate of $\xi$ equals 1 . In general equality holds only if $\operatorname{Char}_{k}(\mathcal{C}) \cap \mathbb{T}^{\xi}=\operatorname{Char}_{k}\left(\mathcal{C}_{\xi}\right)$.

Equation (8) shows that coordinate components have an effect on the topology of the unbranched cover $X_{\rho}$.

As mentioned in the Introduction, Libgober described an algebraic method to compute non-coordinate components. Using deletion of components, this method also exhibits a way to compute non-essential coordinate components. It is still an open problem whether such method exists for essential coordinate components or not. Even though the proof doesn't cover the essential coordinate case, Libgober's method works in certain cases as the following example shows.

Example 4.2. For the sake of simplicity we follow the notation introduced in [13] (Section 2). Consider the sextic curves $\mathcal{C}^{(1)}$ and $\mathcal{C}^{(2)}$ described in the previous section. The contributing ideals of quasiadjunction are described as follows:

(1) $\mathcal{A}_{\left(0, \frac{1}{2}\right)}(1)$. It is supported on $\{Q, R\}$ and its stalks at these points are $\mathfrak{m}_{1}$ and $\mathfrak{m}_{3}$ respectively, where $\mathfrak{m}_{k}=\left(x^{k}, y\right)$ and $(x, y)$ denote a local system of coordinates for which the equation of the singular point $\mathbb{A}_{n}$ is $y^{2}-x^{n+1}$.

(2) $\mathcal{A}_{\left(\frac{1}{2}, 0\right)}(2)$. It is supported on $\{Q, R\}$ and its stalk at both points is $\mathfrak{m}_{3}$.

(3) $\mathcal{A}_{\left(\frac{2}{3}, \frac{1}{6}\right)}(1)$. It is supported on $\{Q, R\}$ and its stalks at these points are $\mathfrak{m}_{2}$ and $\mathfrak{m}_{1}$ respectively. 
The only ideal sheaf with non-trivial irregularity is $\mathcal{A}_{\left(\frac{1}{2}, 0\right)}(2)$ relative to $\mathcal{C}^{(2)}$, due to the existence of the conic $\tilde{\ell}$ passing through three infinitely near points of $Q$ and $R$. As in the essential case, this irregularity describes the existence of a non-trivial point in $\operatorname{Char}_{1}\left(\mathcal{C}^{(2)}\right)$, namely the point $\exp \left(\frac{1}{2}, 0\right)=(-1,1)$.

\section{A TOPOLOGICAL APPROACH}

Finally, we will describe the somewhat surprising difference in the first Betti numbers of unbranched covers of the complement of sextics whose associated branched cover of $\mathbb{P}^{2}$ only ramifies on a conic. First let us give the commutator exact sequences for $G^{(1)}$ and $G^{(2)}$ :

$$
0 \rightarrow\left[G^{(i)}, G^{(i)}\right] \rightarrow G^{(i)} \rightarrow \mathbb{Z} e_{1} \oplus \mathbb{Z} e_{2} \rightarrow 0 .
$$

They were obtained using Reidemeister-Schreier's method. Note that $\left[G^{(i)}, G^{(i)}\right]$ is a cyclic group generated by $t:=\left[e_{1}, e_{2}\right]$ and hence $\left[G^{(i)}, G^{(i)}\right]=M^{(i) \mathbb{Z}}$. The computation gives that $M^{(1) \mathbb{Z}}$ is the cyclic group of order 2 , whereas $M^{(2) \mathbb{Z}}$ is the infinite cyclic group. Moreover, the $\Lambda^{\mathbb{Z}}$-module structure of $M^{(i) \mathbb{Z}}$ is given as follows:

$$
\begin{gathered}
t_{1}\left[e_{1}, e_{2}\right]=e_{1}\left[e_{1}, e_{2}\right] e_{1}^{-1}=\left[e_{1}, e_{2}\right]^{-1}=\left[e_{1}, e_{2}\right] \Rightarrow t_{1} \cdot t=t \\
t_{2}\left[e_{1}, e_{2}\right]=e_{2}\left[e_{1}, e_{2}\right] e_{2}^{-1}=\left[e_{1}, e_{2}\right] \Rightarrow t_{2} \cdot t=t
\end{gathered}
$$

in $M^{(1) \mathbb{Z}}$ and

$$
\begin{gathered}
t_{1}\left[e_{1}, e_{2}\right]=e_{1}\left[e_{1}, e_{2}\right] e_{1}^{-1}=\left[e_{1}, e_{2}\right]^{-1} \Rightarrow t_{1} \cdot t=t^{-1} \\
t_{2}\left[e_{1}, e_{2}\right]=e_{2}\left[e_{1}, e_{2}\right] e_{2}^{-1}=\left[e_{1}, e_{2}\right] \Rightarrow t_{2} \cdot t=t
\end{gathered}
$$

in $M^{(2) \mathbb{Z}}$. This gives the corresponding characteristic varieties over any field.

According to Sakuma's formula in the unbranched case (8), we should look into the branched covering $\rho^{(i)}: \bar{X}_{\rho}^{(i)} \rightarrow \mathbb{P}^{2}$ ramified along the smooth conic $\mathcal{C}_{2}^{(i)}$ in $\mathcal{C}^{(i)}$. It is well known that $\bar{X}_{\rho}^{(i)}$ is isomorphic to $\mathbb{P}^{1} \times \mathbb{P}^{1}$ and the preimage of the conic corresponds to the diagonal curve. A simple local computation shows that the preimage of the quartic curve is a curve of bidegree $(4,4)$ having one $\mathbb{A}_{9}$, one $\mathbb{A}_{5}$ and two $\mathbb{A}_{1}$ points. One can perform a standard Cremona transformation from $\mathbb{P}^{1} \times \mathbb{P}^{1}$ to $\mathbb{P}^{2}$ which sends $\left(\rho^{(i)}\right)^{-1}\left(\mathcal{C}_{4}^{(i)}\right)$ to a sextic curve having four nodes, two $\mathbb{A}_{3}$ points and one $\mathbb{A}_{7}$ point. Such curves must have two irreducible components.

An alternative way to calculate the first Betti number of $X_{\rho}^{(i)}$, the unbranched cover associated with $\rho^{(i)}$, is by direct calculation of the groups. One can compute the fundamental group of $X_{\rho}^{(i)}$ by means of Reidemeister-Schreier's method and thus the rank of its abelianization provides the required Betti number.

Since the Betti number of the complement of curves in $\mathbb{P}^{1} \times \mathbb{P}^{1}$ can be computed from the bidegrees of their irreducible components, one deduces that the two irreducible components of $\left(\rho^{(2)}\right)^{-1}\left(\mathcal{C}_{4}^{(2)}\right)$ have bidegrees $(2,2)$ whereas those of $\left(\rho^{(1)}\right)^{-1}\left(\mathcal{C}_{4}^{(1)}\right)$ have bidegrees $(3,1)$ and $(1,3)$. 


\section{REFERENCES}

1. D. Arapura, Geometry of cohomology support loci for local systems I, J. of Alg. Geom. 6 (1997), 563-597.

2. E. Artal, Sur les couples de Zariski, J. Algebraic Geom. 4 (1994), 223-247.

3. E. Artal, J. Carmona, and J.I. Cogolludo, On sextic curves with big Milnor number, Trends in Singularities (A. Libgober and M. Tibăr, eds.), Trends in Mathematics, Birkhäuser Verlag Basel/Switzerland, 2002, pp. 1-29.

4. E. Artal, J. Carmona, J.I. Cogolludo, I. Luengo, and A. Melle, Fundamental group of plane curves and related invariants, Mathematical contributions: volume in honor of Professor Joaquín Arregui Fernández (Spanish), Editorial Complutense, Madrid, 2000, pp. 77-104.

5. E. Artal, J. Carmona, J.I. Cogolludo, and H.O Tokunaga, Sextics with singular points in special position, J. Knot Theory Ramifications 10 (2001), no. 4, 547-578.

6. G. Barthel, F. Hirzebruch, and T. Höfer, Geradenkonfigurationen und Algebraische Flächen, Friedr. Vieweg \& Sohn, Braunschweig, 1987.

7. J.I. Cogolludo, Topological invariants of the complements to arrangements of rational plane curves, to appear in Memoirs of the AMS.

8. D.C. Cohen and A.I. Suciu, Characteristic varieties of arrangements, Math. Proc. Cambridge Philos. Soc. 127 (1999), no. 1, 33-53.

9. The GAP Group, Aachen, St Andrews, GAP - Groups, Algorithms, and Programming, Version 4.2, 2000, (http://www-gap.dcs.st-and.ac.uk gap).

10. G.-M. Greuel and B. Martin, homolog.lib, A SINGULAR 2.0 library for homological algebra, Centre for Computer Algebra, University of Kaiserslautern, 2001, http://www.singular.uni-kl.de.

11. G.-M. Greuel, G. Pfister, and H. Schönemann, Singular 2.0, A Computer Algebra System for Polynomial Computations, Centre for Computer Algebra, University of Kaiserslautern, 2001, http://www.singular.uni-kl.de.

12. E. Hironaka, Alexander stratifications of character varieties, Ann. Inst. Fourier (Grenoble) 47 (1997), no. 2, 555-583.

13. A. Libgober, Characteristic varieties of algebraic curves, Applications of algebraic geometry to coding theory, physics and computation (Eilat, 2001), Kluwer Acad. Publ., Dordrecht, 2001, pp. 215-254. MR 1866902

14. __ First order deformations for rank one local systems with a non-vanishing cohomology, Topology Appl. 118 (2002), no. 1-2, 159-168, Arrangements in Boston: a Conference on Hyperplane Arrangements (1999).

15. D. Matei, Hall invariants, homology of subgroups and characteristic varieties, Preprint available at arXiv: math.GR/0010046.

16. _ Fundamental groups of links and arrangements: characteristic varieties, resonance varieties, and finite index subgroups, Ph.D. thesis, Northeastern University, Boston, MA, 1999.

17. M. Sakuma, Homology of abelian coverings of links and spatial graphs, Canad. J. Math. 47 (1995), no. 1, 201-224.

18. A.I. Suciu, Fundamental groups of line arrangements: enumerative aspects, Advances in algebraic geometry motivated by physics (Lowell, MA, 2000), Contemp. Math., vol. 276, Amer. Math. Soc., 2001, pp. 43-79. 
Departamento de Matemáticas, Universidad de Zaragoza, Campus Plaza San Francisco S/N, E-50009 ZARAGOZA SPAIN

E-mail address: artal@posta.unizar.es

Departamento de Sistemas informáticos y programación, Universidad Complutense, Ciudad Universitaria s/n, E-28040 Madrid SPAin

E-mail address: jcarmona@eucmos.sim.ucm.es

Departamento de Matemáticas, Universidad de Zaragoza, Campus Plaza San Francisco S/N, E-50009 ZARAGOZA SPAIN

E-mail address: jicogo@posta.unizar.es 